\title{
Why are proton transfers at carbon slow?
}

Cyrille Costentin and Jean-Michel Savéant

\section{Potential energy versus $Q$ profiles}

$\mathrm{CH}_{3} \mathrm{H}+{ }^{-} \mathrm{CH}_{3}$

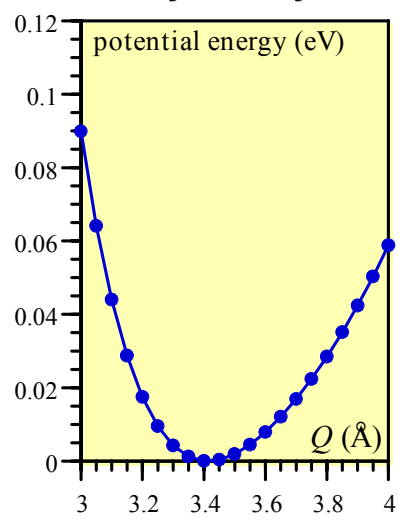

${ }^{+} \mathrm{NH}_{3} \mathrm{H}+\mathrm{NH}_{3}$

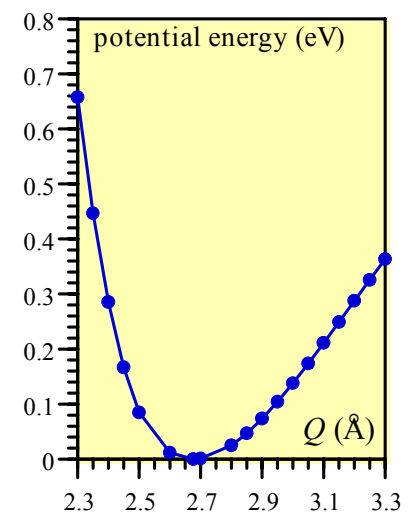

$\mathrm{H}_{2} \mathrm{O}+{ }^{-} \mathrm{OH}$

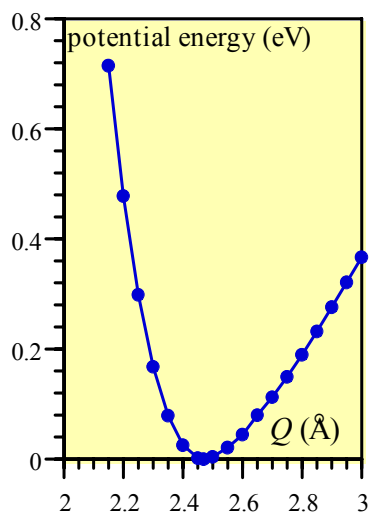

$\mathrm{O}_{2} \mathrm{~N}-\mathrm{CH}_{2}=\mathrm{CH}-\mathrm{CH}_{2} \mathrm{H}+$

$$
\mathrm{CH}_{2}=\mathrm{CH}-\mathrm{CH}=\mathrm{NO}_{2}^{-}
$$

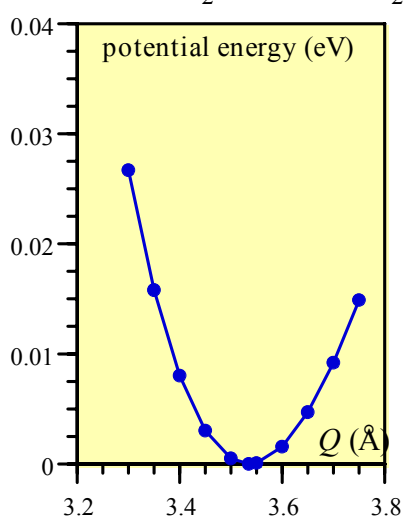

$\mathrm{CH}_{2}=\mathrm{CH}-\mathrm{CH}_{2} \mathrm{H}+{ }^{-} \mathrm{CH}_{2}-\mathrm{CH}=\mathrm{CH}_{2}$

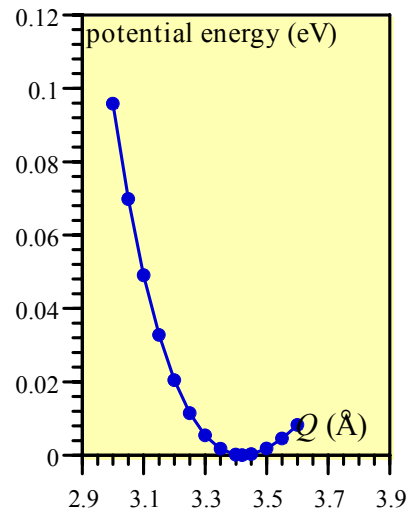

.+

$\stackrel{\bullet+}{\mathrm{H}_{2}} \mathrm{~N}-\mathrm{CH}_{2}=\mathrm{CH}-\mathrm{CH}_{2} \mathrm{H}+$ $\mathrm{CH}_{2}-\mathrm{CH}=\mathrm{CH}_{2}-\mathrm{NH}_{2}$

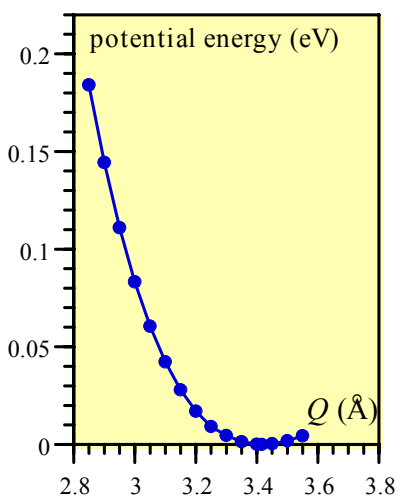

Fig. S1. Potential energy versus $Q$ profiles. B3LYP calculations

QCISD results

\begin{tabular}{|c|c|c|c|c|}
\hline \multicolumn{5}{|c|}{ Table S1. Parameters for the calculation of the rate constants and values of the rate constants. ${ }^{a}$} \\
\hline acid & $\mathrm{CH}_{4}$ & $\mathrm{H}_{2} \mathrm{O}$ & $\mathrm{CH}_{2}=\mathrm{CH}-\mathrm{CH}_{3}$ & \multirow{14}{*}{$\begin{array}{l}\text { a: force constants in } \mathrm{eV} \AA^{-2} \text {, energies in } \mathrm{eV} \text {, } \\
\text { distances in } \AA \text {. b: } f=(2 \pi \mu v)^{2} \text {. c: } v_{Q} \text { from the } \\
\text { potential energy vs. } Q \text { diagrams in the supporting } \\
\text { information; } \mu=6 \mathrm{amu} \text { except for } \mathrm{H}_{2} \mathrm{O} \text { where } \mu=8 \\
\text { amu. } \mathrm{d} \text { : } v_{0}, v_{b} \text { from the potential energy vs. } q \\
\text { diagrams in figures } \mathrm{S} 3 ; \mu=1 \mathrm{amu} \text {. e: from equation } \\
\text { (4) taking }\left(e_{0}^{2} / 4 \pi \varepsilon_{0}\right)\left(1 / \varepsilon_{o p}-1 / \varepsilon_{S}\right)=4 \text { as derived } \\
\text { from experimental values found in the application of } \\
\text { Hush-Marcus theory to homogeneous self-exchange } \\
\text { reaction in DMF, la rather than direct application of } \\
\text { the Born-like Marcus formula expected to } \\
\text { overestimate solvation energies as discussed in } \\
\text { reference } 1 \mathrm{~b} \text {. f: from the potential energy vs. } q \\
\text { diagrams in figures } \mathrm{S} 2 \text {. g: from equation }(3) \text {, taking } \\
a_{R}=a_{P}=2 \AA \text {. h: from equation }(7) \text {, taking } \beta=30 \AA^{-} \\
1 \text { : bimolecular rate constant in } \mathrm{M}^{-1} \mathrm{~s}^{-1} \text { derived } \\
\text { according to the procedure described in the text with } \\
Z / v=0.05 \mathrm{M}^{-1} \text {. }\end{array}$} \\
\hline$f_{Q}^{\mathrm{b}, \mathrm{c}}$ & 0.638 & 3.854 & 0.655 & \\
\hline$f_{0}{ }^{\mathrm{b}, \mathrm{d}}$ & 19.2 & - & 20.4 & \\
\hline$f_{b}{ }^{\mathrm{b}, \mathrm{d}}$ & 5.93 & - & 8.47 & \\
\hline$h v_{Q}{ }^{\mathrm{c}}$ & 0.021 & 0.045 & 0.0215 & \\
\hline$h v_{0}{ }^{d}$ & 0.283 & - & 0.292 & \\
\hline$h v_{b}{ }^{\mathrm{d}}$ & 0.157 & - & 0.188 & \\
\hline$Q_{0}{ }^{\mathrm{c}}$ & 3.543 & 2.49 & 3.537 & \\
\hline$Q^{\neq \mathrm{e}}$ & 3.409 & 2.45 & 3.405 & \\
\hline$\Delta V^{\mathrm{f}}$ & 0.874 & 0.0 & 0.89 & \\
\hline$\lambda_{0}\left(Q^{\neq}\right)^{\mathrm{g}}$ & 0.827 & 0.366 & 0.825 & \\
\hline$\lambda_{i}$ & 0.216 & 0.084 & 0.65 & \\
\hline$E_{\beta}{ }^{\mathrm{h}}$ & 0.314 & 0.235 & 0.314 & \\
\hline $\log k^{\mathrm{i}}$ & -4.74 & 9.6 & -5.21 & \\
\hline
\end{tabular}

1. (a) Kojima, H.; Bard, A. J. J. Am. Chem. Soc. 1975, 77, 5317. (b) Andrieux, C. P.; Savéant, J-M.; Tardy, C. J. Am. Chem Soc. 1998, $120,4167$. 

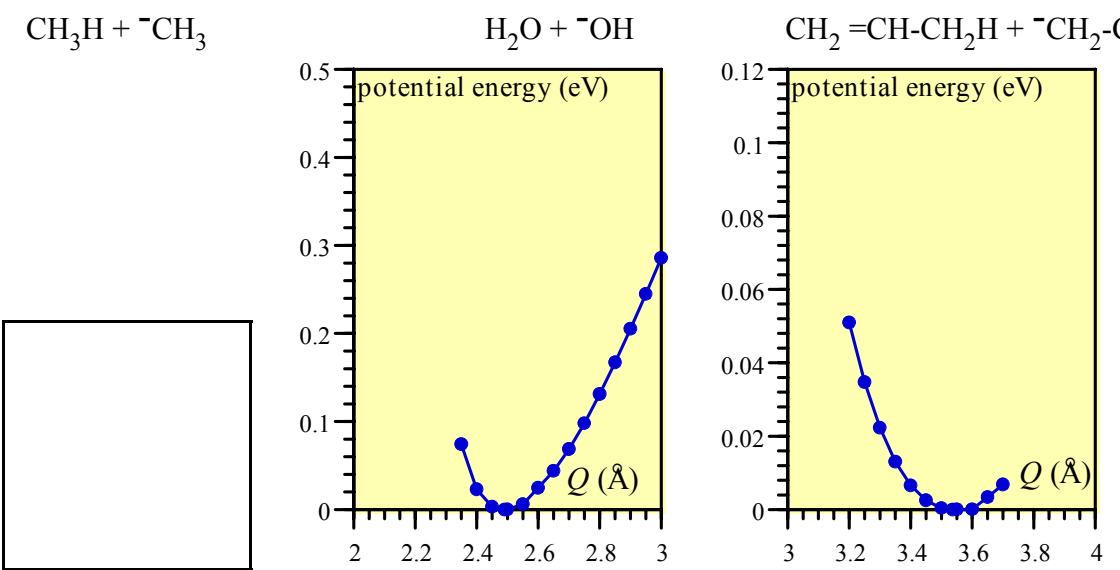

Fig. S2. Potential energy versus $Q$ profiles. QCISD calculations
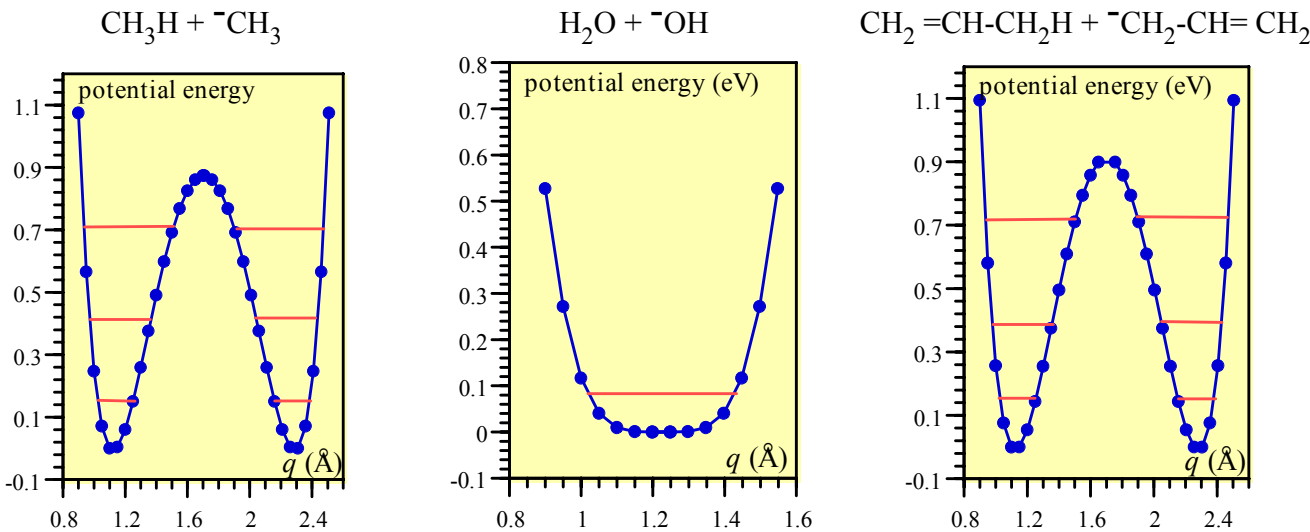

Fig. S3. Potential energy versus $q$ profiles for $Q=Q^{\neq}$. QCISD calculations 